\title{
Geoestatística aplicada na estimativa de chuvas máximas diárias no estado de Minas Gerais
}

\author{
Rafael Alvarenga ALMEIDA ${ }^{1 *}$, Gilberto COELHO², Isabela Alvarenga ALMEIDA³, \\ Jéfferson de Oliveira COSTA ${ }^{3}$
}

\author{
${ }^{1}$ Universidade Federal dos Vales do Jequitinhonha e Mucuri, Mucuri, MG, Brasil. \\ ${ }^{2}$ Departamento de Engenharia Agrícola, Universidade Federal de Lavras, Lavras, MG, Brasil. \\ ${ }^{3}$ Programa de Pós-Graduação em Engenharia de Sistemas Agrícolas, Escola de Agricultura Luiz de Queiroz, \\ Universidade de São Paulo, Piracicaba, SP, Brasil \\ *E-mail: rafael.almeida@ufvjm.edu.br
}

Recebido em novembro/2017; Aceito em agosto/2018.

\begin{abstract}
RESUMO: O objetivo desse trabalho foi determinar os parâmetros $\alpha$ e $\beta$ espacializados e, assim, possibilitar a estimativa de chuvas intensas em todo o estado de Minas Gerais com uma determinada probabilidade, e também para tempos de retorno diferentes dos tradicionais. A área de estudo considerada foi o Estado de Minas Gerais, foram utilizados dados de 96 estações pluviométricas presentes na região e aplicados testes de aderência da distribuição generalizada de valores extremos (GEV) e de tendência. Os resultados da avaliação da dependência espacial dos parâmetros $\alpha$ e $\beta$ e seus respectivos intervalos de confiança, demonstram que o modelo de semivariograma exponencial foi o mais adequado para descrever a dependência espacial dos parâmetros da distribuição GEV, podendo ser aplicados os valores de precipitação máxima diária no Estado de Minas Gerais. Concluiu-se que os mapas gerados podem ser utilizados para determinação dos parâmetros $\alpha$ e $\beta$ em qualquer localidade de Minas Gerais e, assim, a precipitação máxima diária pode ser obtida para quaisquer tempos de retorno desde que os intervalos de confiança de $\alpha$ e $\beta$ sejam respeitados.
\end{abstract}

Palavras-chave: eventos extremos, precipitação máxima, probabilidade.

\section{Geostatistics applied in the estimation of daily maximum rains in the state of Minas Gerais}

\begin{abstract}
The objective of this work is to determine the parameters $\alpha$ and $\beta$ estimates of rainfall in the entire state of Minas Gerais with a certain probability, as well as our traditional return times. A study area of the State of Minas Gerais, with rainfall data of 96 rainfall stations present in the region and tested for generalized distribution of extreme values (GEV)adherence and trend. The results of the spatial dependence evaluation of the parameters and their confidence parameters demonstrate that the exponential semivariogram model was the most adequate to describe a spatial dependence of the parameters of the GEV distribution, being able to be applied to values of daily maximum non-state precipitation Minas Gerais. It was concluded that the generated maps can be used for the determination of the parameters and in any locality of Minas Gerais and, thus, a maximum daily precipitation, can be obtained by times of return since the confidence intervals of $\alpha$ and $\beta$ are respected.
\end{abstract}

Keywords: extreme events, maximum precipitation, probability.

\section{INTRODUÇÃO}

A chuva pode ser considera a principal forma de suprimento hídrico para as atividades humanas e econômicas, principalmente na agricultura, setor em que a precipitação é usada, direta ou indiretamente, em processos de tomada de decisão, como por exemplo: no manejo da irrigação, no risco de incêndios, na previsão de geadas, no zoneamento agroclimático, na ocorrência de pragas e doenças, na classificação climática, entre outros (ALMEIDA et al., 2011).

Ainda de acordo Almeida et al. (2011), a precipitação é,dentre os parâmetros meteorológicos, a que apresenta a maior variabilidade temporal e espacial sendo, portanto, o de maior dificuldade de estimativa. Com isto, tornam- se fundamentais a caracterização do seu padrão local e suas variações.

Tucci (2000) afirma que o conhecimento da precipitação máxima é fundamental para a determinação da vazão de enchente de uma bacia hidrográfica, considerando maior a disponibilidade de longas séries de precipitações comparada as séries históricas de vazão. Segundo o mesmo autor, o escoamento superficial é o responsável na maioria das vezes, pela ocorrência de alagamentos, considerado a parcela do ciclo hidrológico em que a água se desloca na superfície da bacia até encontrar uma drenagem definida. Neste aspecto, quando a bacia hidrográfica é constituída por cobertura vegetal, o escoamento sofre a interferência desta, e uma parte dele se infiltra. Entretanto se a bacia hidrográfica se encontra impermeabilizada, como em áreas urbanas, essa parcela não se infiltra gerando grandes vazões.

Segundo Mello et al. (2008), a análise de chuvas intensas consiste em um dos produtos mais aplicados da hidrologia. Seu uso está associado ao dimensionamento de obras hidráulicas para controle de cheias, abastecimento e condução de água para usos múltiplos, drenagem urbana e do solo e também para modelagem e controle da erosão.

De acordo Mello; Silva (2009), em diversos trabalhos foram analisadas a dependência espacial da precipitação, que é uma técnica comumente empregada nesse tipo de estudo. Segundo Almeida et al. (2011) a geoestatística parte do princípio de que há forte correlação entre observações no 
espaço, e oferece ferramentas determinísticas e estatísticas que possibilitam entender e modelar a variabilidade espacial da precipitação. No que tange à dependência temporal, poucas são as análises realizadas com essa metodologia.

Apesar da teoria probabilística fundamental de valores extremos ter sido desenvolvida há muito tempo, a modelagem estatística de extremos ainda permanece como assunto ativo de pesquisas dado seu importante papel nos projetos e gerenciamento de recursos hídricos, especialmente num contexto de mudanças climáticas (KATZ et al., 2002).

O mapeamento de variáveis climáticas, como chuvas intensas, é fundamental para o manejo ambiental e agrícola. Para isto, ferramentas estatísticas para interpolação espacial devem ser devidamente analisadas e caracterizadas (MELLO et al., 2008; LIMA et al., 2008).

O objetivo desse trabalho foi determinar os parâmetros $\alpha$ e $\beta$ da GEV espacializados e, assim, possibilitar a estimativa de chuvas intensas em todo o estado de Minas Gerais com uma determinada probabilidade, para diferentes tempos de retorno.

\section{MATERIAL E MÉTODOS}

A Figura 1 apresenta na forma de um fluxograma as etapas que foram desenvolvidas. Em primeiro momento foram elencadas todas as estações pluviométricas situadas no Estado de Minas Gerais junto ao sistema Hidroweb/ANA. Elencou-se as que possuíam uma série de dados com mais de 30 anos de dados, para que pudessem ser organizados em planilhas eletrônicas.

As próximas etapas estão melhor descritas na sequência e consistiram sequencialmente: 1) aplicação do teste de aderência das séries históricas à distribuição de probabilidades; 2) aplicação do teste de análise de tendência nas séries históricas; 3) aplicação da distribuição de probabilidades GEV e obtenção dos parâmetros $\alpha$ e $\beta$, bem como seus respectivos intervalos de confiança; 4) análise das estruturas de dependência espacial dos parâmetros; e 5) Espacialização dos parâmetros e seus intervalos de confiança.

\subsection{Localização e caracterização da área de estudo}

A área de estudo considerada foi o Estado de Minas Gerais que é considerado a quarta maior unidade federativa do Brasil em extensão territorial $\left(586.528 \mathrm{~km}^{2}\right)$ e o terceiro em população. Está localizado na Região Sudeste do Brasil, limita-se ao sul e sudoeste com o estado de São Paulo e a oeste com o estado de Mato Grosso do Sul e a noroeste com Goiás, incluindo uma pequena divisa com o Distrito Federal, a leste com o Espírito Santo, a sudeste com o Rio de Janeiro e a norte e nordeste com a Bahia.

O Estado apresenta uma condição topográfica muito variada e, segundo a classificação de Köppen, são encontrados os seguintes tipos de clima: Aw, BSw, Cwa e Cwb, significando que existe uma grande diversidade climática, podendo ser encontradas desde regiões com clima semi-árido até regiões com clima tropical chuvoso com inverno seco.

$\mathrm{O}$ índice pluviométrico em Minas Gerais é em média $1.500 \mathrm{~mm}$ ano $^{-1}$, sendo que a estação de chuva é o verão, quando a massa equatorial continental está sobre a região. No inverno, com o deslocamento dessa massa, diminui a umidade e então ocorre a estação seca. Na Figura 2 pode ser observada a distribuição espacial das 96 estações pluviométricas, que dispunham de uma série histórica superior a 30 anos e foram utilizadas neste estudo.

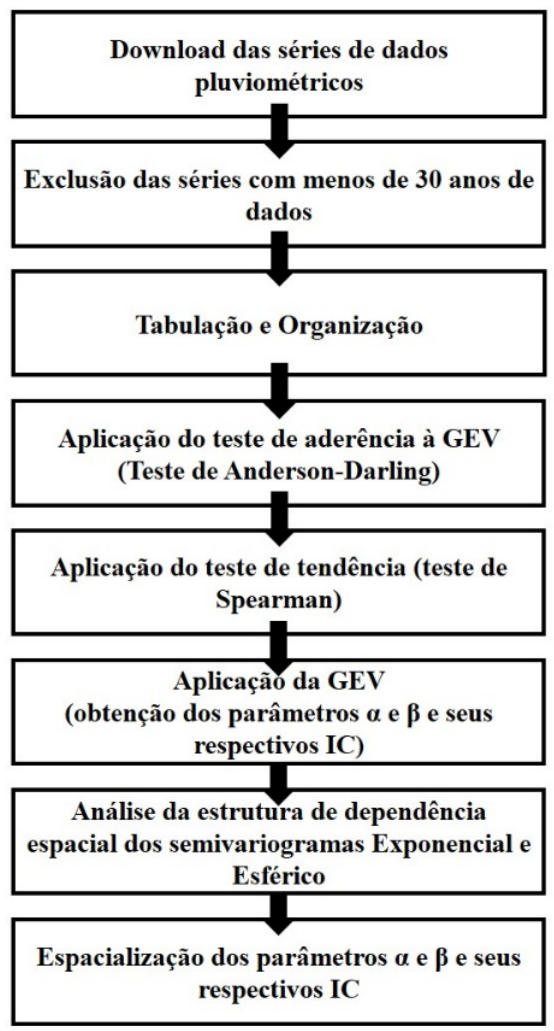

Figura 1. Fluxograma com as etapas para o desenvolvimento do trabalho.

Figure 1. Flow chart with the steps for the development of the work.

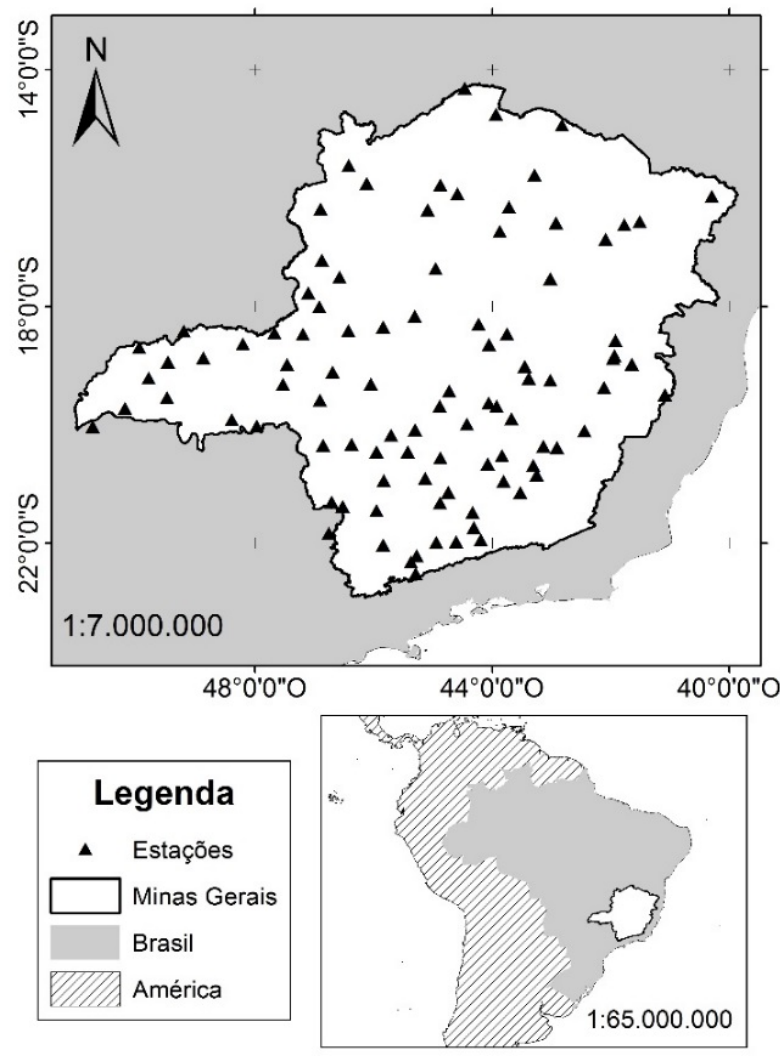

Figura 2. Localização das estações pluviométricas.

Figure 2. Location of pluviometric stations. 


\subsection{Teste de aderência}

Para verificar a adequabilidade da distribuição GEV aos dados amostrais, foram realizadas comparações entre as frequências amostrais e as frequências teóricas esperadas utilizando um modelo probabilístico para descrever os dados observados. Com os resultados dessas comparações verificou-se uma possível explicação da distribuição de probabilidades ou não.

Foi utilizado o teste de Anderson-Darling para a verificação da aderência dos dados à GEV, que pode ser descrito com clareza por Naghettini; Pinto (2007). Este teste é uma modificação do teste Kolmogorov-Smirnov, o qual utiliza a distribuição específica, na hipótese nula, para calcular os quantis críticos do teste. Se aplica melhor a GEV, pois este é um teste que independe do número de classes e tem grande aplicabilidade em situações onde os dados possuem assimetria, ou seja teste de valores extremos (NAGHETTINI; PINTO, 2007).

\subsection{Teste de análise de tendência}

Foi utilizado o teste Spearmam, o qual é tido como uma técnica rigorosa, extremamente eficiente e que tem aplicação frequente para análise da estacionariedade de séries hidrológicas e pode ser descrito por Naghettini; Pinto (2007).

$\mathrm{O}$ teste referido possibilitou a observação da ocorrência de estacionaridade da série, ou seja, se a mesma apresenta uma tendência temporal. Para 0 caso de variáveis hidrometeorológicas, esse teste tem a possibilidade de detectar possíveis tendências temporais nas séries históricas. Os resultados desses estudos tornam-se fundamentais para o planejamento dos recursos hídricos de uma região.

2.4. Aplicação da distribuição generalizada de valores extremos (GEV) e geração dos mapas temáticos

Após a aplicação dos testes estatísticos de análise de tendências e o de aderência da série histórica, realizou-se a distribuição generalizada de valores extremos (GEV) utilizando o software Excel ${ }^{\circledR}$, para o cálculo dos parâmetros $\alpha$ e $\beta$, e seus respectivos intervalos de confiança (IC) para cada estação. Para a estimativa dos parâmetros da distribuição GEV, foi utilizado o método da máxima verossimilhança (MMV) conforme o trabalho de Villarini (2011) e para estimativa dos intervalos de confiança, foi utilizada a metodologia descrita em Naghettini; Pinto (2007).

A estrutura de dependência espacial dos parâmetros $\alpha \mathrm{e}$ $\beta$ da distribuição GEV e os respectivos intervalos de confiança (IC) foram adequados a dois modelos de semivariograma, esférico e exponencial, com o intuito de descrever o comportamento da distribuição espacial da precipitação máxima diária no Estado de Minas Gerais. O método de ajuste dos modelos de semivariograma utilizado foi o método dos Mínimos Quadrados. Como critério de escolha do melhor modelo, considerou-se o grau de dependência espacial (GD) e o alcance espacial do modelo utilizado.

Após ter sido selecionado o melhor modelo de semivariograma, foi utilizado o método da krigagem para interpolação dos dados e, posteriormente, foram elaborados mapas temáticos dos parâmetros da distribuição GEV e seus respectivos intervalos de confiança (IC). Para a modelagem dos semivariogramas e geração dos mapas, utilizou-se o aplicativo ArcMap, do software ArcGis Desktop 10.5®.

\section{RESULTADOS}

Os resultados obtidos demonstraram que das 96 séries históricas analisadas, $14,6 \%$ não se adequaram a distribuição GEV segundo o teste de Anderson-Darling. Já para o teste de Spearman, 16,7\% apresentaram tendências nas séries históricas de precipitações máximas diárias. As estações que não são adequadas pelo teste de Spearman, não apresentam tendências regionais, em função da sua distribuição espacial. Dentre todas as séries, apenas 7,3\% apresentaram problemas de aderência e tendência simultaneamente (Figura 3).

Quanto ao parâmetro $\alpha$ da distribuição GEV, o modelo exponencial apresentou melhor alcance $(526,41 \mathrm{~km})$ e melhor grau de dependência espacial $(34,9 \%)$, sendo considerado moderado este grau de dependência espacial (Tabela 1).

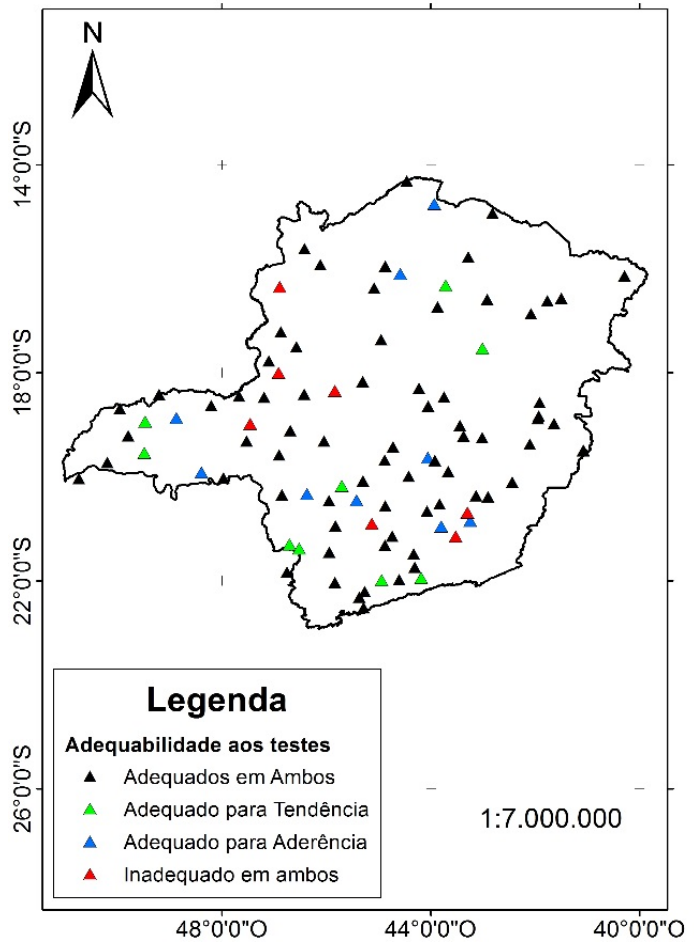

Figura 3. Resultados dos testes estatísticos.

Figure 3. Statistical test results.

Além disso, verifica-se que para o parâmetro $\beta$, o modelo exponencial e o esférico apresentaram o mesmo alcance $(1072,57 \mathrm{~km})$, porém, o modelo exponencial apresenta um melhor grau de dependência espacial (51,7\%), também classificado como moderado grau de dependência espacial (Tabela 1)

Tabela 1. Efeito pepita (C0), Variância estruturada (C1), Alcance e Grau de dependência espacial (GD), referentes ao parâmetro $\alpha$ e $\beta$.

Table 1. Effect putative (C0), Structured Variance (C1), Range and Degree of spatial dependence (GD), referring to parameter $\alpha$ and $\beta$.

\begin{tabular}{ccccc}
\hline Parâmetro $\alpha$ & $\mathrm{C} 0$ & $\mathrm{C} 1$ & Alcance $(\mathrm{km})$ & $\mathrm{GD}$ \\
\hline Mod. Exponencial & 8,84 & 4,73 & 526,41 & 34,9 \\
Mod. Esférico & 12,6 & 28,0 & 435,79 & 30,7 \\
\hline Parâmetro $\beta$ & $\mathrm{C} 0$ & $\mathrm{C} 1$ & Alcance $(\mathrm{km})$ & $\mathrm{GD}$ \\
\hline Mod. Exponencial & 28,28 & 30,22 & 1072,57 & 51,7 \\
Mod. Esférico & 31,71 & 30,01 & 1072,57 & 48,6 \\
\hline
\end{tabular}

$\mathrm{Na}$ Figura 4A pode-se observar a distribuição espacial do parâmetro $\alpha$, utilizando o modelo de semivariograma exponencial. Verifica-se que os maiores valores se encontram 
nas Regiões Sul (região da Serra da Mantiqueira) e Noroeste de Minas. Enquanto isso, os menores valores se encontram na Região Nordeste do Estado, nas proximidades do Rio Jequitinhonha.

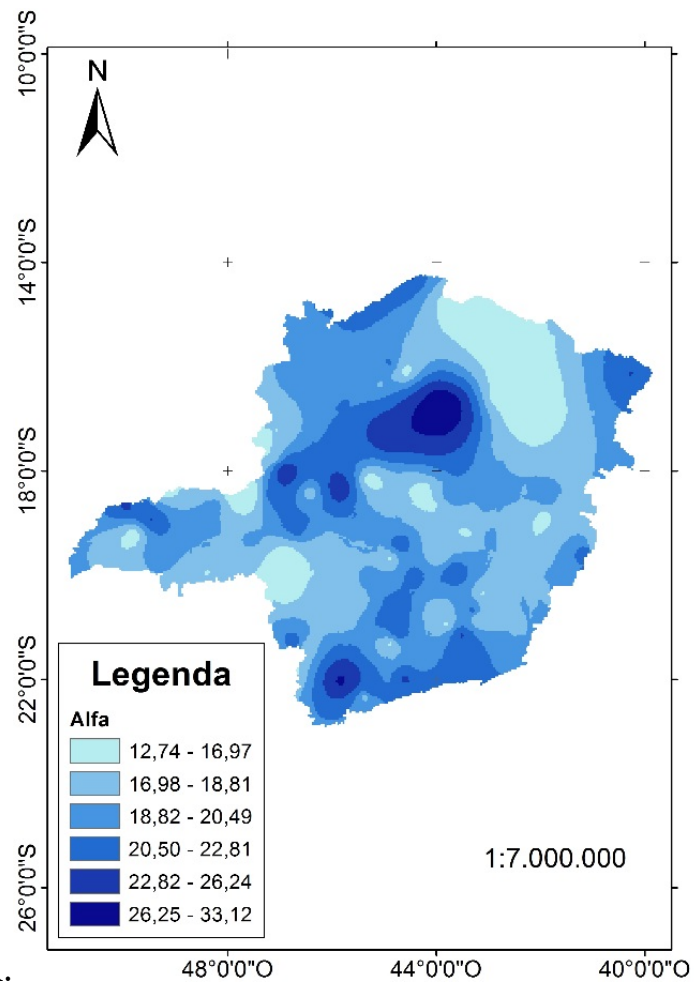

A.

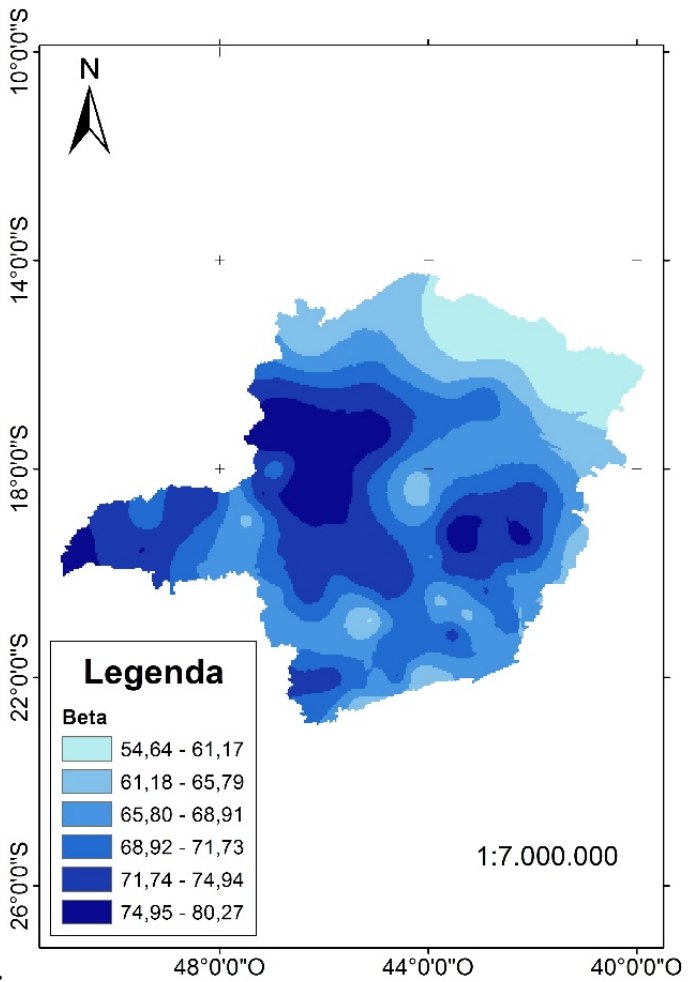

Figura 4. Distribuição espacial dos parâmetros $\alpha$ (A) e $\beta$ (B) para o Estado de Minas Gerais.

Figure 4. Spatial distribution of the parameters $\alpha(A)$ and $\beta$ (B) for the State of Minas Gerais.

Com relação a parâmetro $\beta$ (Figura $4 \mathrm{~B}$ ), os maiores valores foram encontrados na extremidade do Triângulo Mineiro e na Região Noroeste e, semelhante ao parâmetro $\alpha$, na região Nordeste predominaram os menores valores.
Quanto aos intervalos de confiança, o modelo esférico apresentou o maior alcance tanto para o intervalo de confiança de $\alpha$ como para o de $\beta$ (Tabela 2), sendo o alcance igual a $333,18 \mathrm{~km}$ para ambos os intervalos de confiança. Entretanto, o modelo exponencial foi o que apresentou melhor grau de dependência espacial $(47,9 \%)$ para os dois intervalos de confiança.

Tabela 2. Efeito pepita (C0), Variância estruturada (C1), Alcance e Grau de dependência espacial (GD), referentes ao intervalo de confiança do parâmetro $\alpha$ e $\beta$.

Table 2. Effect putative $(\mathrm{C} 0)$, structured Variance $(\mathrm{C} 1)$, Range and Degree of spatial dependence (GD), referring to the confidence interval of parameter $\alpha$ and $\beta$.

\begin{tabular}{ccccc}
\hline Parâmetro $\alpha$ & $\mathrm{C} 0$ & $\mathrm{C} 1$ & $\begin{array}{c}\text { Alcance } \\
(\mathrm{km})\end{array}$ & $\mathrm{GD}(\%)$ \\
\hline $\begin{array}{c}\text { Modelo } \\
\text { Exponencial } \\
\text { Modelo } \\
\text { Esférico }\end{array}$ & 0,75 & 0,69 & 258,96 & 47,9 \\
\hline Parâmetro $\beta$ & 0,97 & 0,48 & 333,18 & 33,1 \\
\hline $\begin{array}{c}\text { Modelo } \\
\text { Exponencial } \\
\text { Modelo } \\
\text { Esférico }\end{array}$ & 1,37 & 1,26 & 258,96 & 47,9 \\
\hline
\end{tabular}

$\mathrm{Na}$ Figura 5A observa-se a distribuição espacial do intervalo de confiança do parâmetro $\alpha$ e na Figura 5B do parâmetro $\beta$, utilizando o modelo de semivariograma exponencial. Verifica-se que os dois mapas apresentam comportamento semelhante, com pequena amplitude de variação em ambas as situações.

\section{DISCUSSÃO}

Os resultados da avaliação de dependência espacial dos parâmetros $\alpha$ e $\beta$ e seus respectivos intervalos de confiança, demonstram que o modelo de semivariograma exponencial foi o mais adequado para descrever a dependência espacial dos parâmetros da distribuição GEV, podendo ser aplicado a valores de precipitação máxima diária no estado de Minas Gerais. Diversos autores, em estudos com objetivo semelhantes estabeleceram também outras metodologias para a determinação das chuvas extremas em outras localidades. Tais estudos corroboram para a importância da determinação de chuvas intensas.

Machado et al. (2010) avaliou a distribuição das precipitações pluviais máximas diárias anuais para distintos períodos de retorno por meio de técnicas geoestatísticas para o Rio de Janeiro. As séries de dados foram procedentes de 119 postos pluviométricos, apresentando em média 36 anos de observações. Em cada estação foi identificada a chuva máxima diária anual, posteriormente foram estimados os eventos associados a diferentes períodos de retorno por meio da distribuição Gumbel, e com esses dados, obtiveram-se os modelos experimentais de semivariogramas. Mostrando que os mapas interpolados foram mais erráticos em períodos de retorno mais altos, ao Noroeste, Norte e em regiões de planície costeira, recomendando-se novas instalações de estações pluviométricas. Cabe comparar, que este estudo contou com uma maior quantidade de estações e um maior período de dados observados, tais fatos podem caracterizar uma maior confiabilidade nos dados. Outro fator a ser observado diz respeito à uma necessidade de ampliação das 
estações pluviométricas no Estado de Minas Gerais, que conta com um número bem menor de estações mesmo que com uma área superior ao do Estado do Rio de Janeiro.

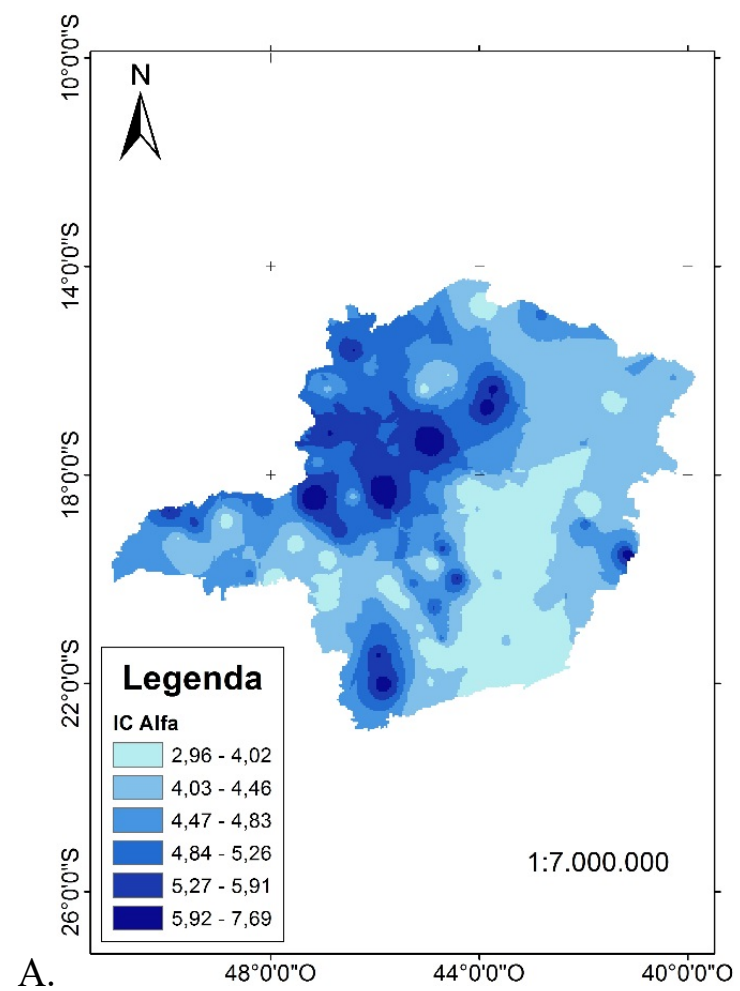

A.

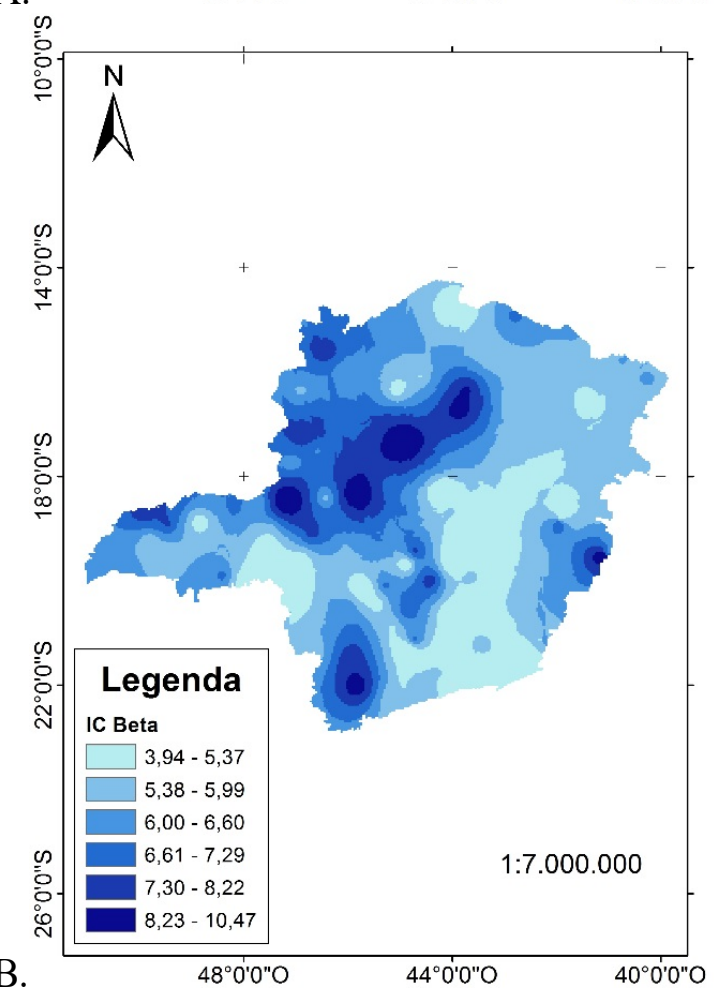

Figura 5. Distribuição espacial do intervalo de confiança dos parâmetros $\alpha$ (A) e $\beta$ (B) para o Estado de Minas Gerais.

Figure 5. Spatial distribution of the confidence interval of the parameters $\alpha(\mathrm{A})$ and $\beta$ (B) for the State of Minas Gerais.

Beijo; Avelar (2010) expuseram uma revisão a respeito da utilização da distribuição generalizada de valores extremos em dados ambientais e apresentaram sua aplicação com enfoque clássico e bayesiano no estudo de precipitação máxima ajustando-a aos dados de Carbonita-MG. Demonstraram também a grande importância para a modelagem de dados climáticos extremos e apropriada aos dados de precipitações máximas, necessitando a ampliação de estudos semelhantes em outras regiões.

Freire et al. (2013), determinaram, através da distribuição de Gumbel, as precipitações máximas para diferentes períodos de retorno em São João do Jaguaribe-CE. O método mostrou-se adequado, satisfatório e preciso para estimativa das precipitações máximas no município, representando uma alternativa na determinação das chuvas máximas de projetos, além do método dos momentos ter se mostrado como uma maneira rápida de aquisição de dados, possibilitando maior rapidez na análise dos resultados. Mesmo com a utilização de uma outra distribuição de probabilidades, as chuvas intensas necessitam de uma estimativa. Tal fato pode ser explicado pelas diferentes características dos regimes pluviométricos que governam o Brasil.

Oliveira et al. (2008) desenvolveram uma rotina computacional para ajustar os parâmetros do modelo de Bell para municípios do estado de Goiás, com propósito de estimar alturas de chuvas intensas associadas a uma duração e a uma frequência. Utilizaram séries históricas de precipitações diárias e de relações intensidade-duraçãofrequência disponíveis, além da relação entre a precipitação de 60 minutos e 1 dia de duração, para um período de retorno de dois anos. O modelo de Bell ajustou-se perfeitamente para os municípios estudados, apresentando alternativa na obtenção das alturas de chuvas intensas a partir de séries curtas. A reconstrução do modelo a partir de regionalização dos parâmetros concedeu a ampliação das equações que apresentam a relação entre a precipitação máxima para diferentes durações e o período de retorno com baixos valores no erro-padrão da estimativa.

Mello et al. (2001) ajustaram o modelo exponencial, linear e básico para estimativa de chuvas intensas. Os modelos foram ajustados com base em dados de precipitação máxima diária anual da região de Lavras-MG, em um período de 1914 a 1991. Os dados foram transformados em intensidade de precipitação, com variação do tempo de retorno de 2 a 100 anos e duração entre 5 e 1.440 minutos. Constataram que o modelo exponencial proporcionou ajustes melhores, com menores erros na estimativa, para chuvas variando de 5 a $240 \mathrm{~min}$, sendo aconselhável a bacias em que o tempo de concentração se ajuste nesta faixa de tempo. O modelo básico se mostrou mais aplicável a bacias em que o tempo de duração seja maior que $240 \mathrm{~min}$, enquanto o modelo linear não se mostrou confiável para a estimativa de chuvas intensas. Pode-se observar uma semelhança no fato do modelo exponencial obter um melhor desempenho na estimativa de chuvas intensas no Estado de Minas Gerais, tal fato corrobora com os resultados obtidos.

Hartmann et al. (2011) estimaram a precipitação pluvial máxima esperada para diferentes níveis de probabilidade e verificaram o grau de ajuste dos dados ao modelo de Gumbel, com as estimativas dos parâmetros adquiridas pelo método de máxima verossimilhança na região de Presidente Prudente. Os resultados apresentam um bom ajuste na distribuição Gumbel para os dados da precipitação máxima mensal para a região em estudo e as estimativas de precipitação adquiridas pelo método de máxima verossimilhança são consistentes reproduzindo com alta fidelidade o regime de chuvas da região. 
Genovez; Zuffo (2010) apresentaram uma revisão sobre os estudos da relação Intensidade-Duração- Frequência (IDF) de chuva para o estado de São Paulo. Os estudos compreendem as equações IDF locais e generalizadas, as relações entre as chuvas intensas de diferentes durações e os mapas de isoietas. De acordo com os autores, métodos que se baseiam nas relações de chuvas intensas de variações diferentes têm valia regional e que para estimativas locais é pertinente determinar novos coeficientes relacionados às características locais dos microclimas.

Ainda, na literatura apresentada, são observados os valores para os tempos de retorno mais comuns $(5,10,20,50$ e 100 anos). Com a espacialização dos parâmetros de uma distribuição de probabilidades adequada a grande maioria das estações, torna-se possível a estimativa de chuvas intensas com qualquer tempo de retorno e não apenas os mais comumente utilizados.

\section{CONCLUSÕES}

Concluiu-se que a distribuição GEV pode ser aplicada para descrever o comportamento das séries históricas de precipitações máximas diárias do estado de Minas Gerais, e que a grande maioria das séries históricas analisadas não apresentaram mudanças no comportamento temporal de ocorrência de precipitações máximas diárias.

$\mathrm{O}$ modelo exponencial mostrou-se mais adequado no ajuste dos parâmetros da distribuição GEV para precipitações máximas diárias no Estado de Minas Gerais, podendo-se ser empregado para espacialização dos dados por meio de interpolação geoestatística.

Os mapas gerados podem ser utilizados para determinação dos parâmetros $\alpha$ e $\beta$ em qualquer localidade de Minas Gerais e, assim, a precipitação máxima diária pode ser obtida para quaisquer tempos de retorno desde que os intervalos de confiança de $\alpha$ e $\beta$ sejam respeitados.

\section{AGRADECIMENTOS}

Agradecimentos a Universidade Federal dos Vales do Jequitinhonha e Mucuri, a Universidade Federal de Lavras e a Universidade de São Paulo pelo apoio à pesquisa e aliança realizada.

\section{REFERÊNCIAS}

ALMEIDA, A. Q. de; RIBEIRO, A.; PAIVA, Y. G.; RASCON, N. J. L.; LIMA, E. D. P. Geoestatística no estudo de modelagem temporal da precipitação. Revista Brasileira de Engenharia Agrícola e Ambiental, Campina Grande, v. 15, n. 4, p. 354-358, 2011. DOI: http://dx.doi.org/10.1590/S1415-43662011000400004

BEIJO, L. A.; AVELAR, F. G. Distribuição Generalizada de Valores Extremos no estudo de dados climáticos: uma breve revisão e aplicação. Revista da Estatística UFOP, Ouro Preto, v. 1, n. 1, p. 10-16, 2010.

FREIRE, F. G. C.; BARRETO, H. B. F.; BATISTA, R. O.; SANTOS, W. de O. Estudo das precipitações máximas para diferentes períodos de retorno pela distribuição de gumbel no município de São João do Jaguaribe/CE. Revista brasileira de agricultura irrigada, Fortaleza, v. 7, n. 5, p. 300-305, 2013. DOI: http://dx.doi.org/10.7127/RBAI.V7N500169

GENOVEZ, A. M.; ZUFFO, A. C. Chuvas intensas no Estado de São Paulo: Estudos existentes e análise comparativa. Revista Brasileira de Recursos Hídricos,
Porto Alegre, v. 5, n. 3, p. 45-58, 2000. DOI: http://dx.doi.org/10.21168/rbrh.v5n3.p45-58

HARTMANN, M.; MOALA, F. A.; MENDONÇA, M. A. Estudo das precipitações máximas anuais em Presidente Prudente. Revista Brasileira de Meteorologia, São Paulo, v., 26, n. 4, p. 561-568, 2011. DOI: http://dx.doi.org/10.1590/S0102-77862011000400006

KATZ, R. W.; PARLANGE, M. B.; NAVEAU, P. Statistics of extremes in hydrology. Advances in Water Resources, Southampton, v. 25, n. 8-12, p. 1287-1304., 2002. DOI: https://doi.org/10.1016/S03091708(02)00056-8

LIMA, J. S. S.; SILVA, S. A.; OLIVEIRA, R. B.; CECÍLIO, R. A.; XAVIER, A. C. Variabilidade temporal da precipitação mensal em Alegre - ES. Revista Ciência Agronômica, Fortaleza, v. 39, n. 2, p. 327-332, 2008.

MACHADO, R.L.; CEDDIA, M. B.; CARVALHO, D. F. D.; CRUZ, E. S. D.; FRANCELINO, M. R. Spatial variability of maximum anual daily rain under diferente return periods at the Rio de Janeiro state, Brazil. Bragantia, Campinas, v. 69, p. 77-84, 2010. DOI: http://dx.doi.org/10.1590/S0006-87052010000500009

MELLO, C. R. de; FERREIRA, D. F.; SILVA, A. M.; LIMA, J. M. Análise de modelos matemáticos aplicados ao estudo de chuvas intensas. Revista brasileira de ciência do solo, Viçosa, v. 25, n. 3, p. 693-698, 2001. DOI: http://dx.doi.org/10.1590/S0100-06832001000300018

MELlO, C. R. de; VIOLA, M. R.; MELLO, J. M. de; SILVA, A. M. da. Continuidade espacial de chuvas intensas no estado de Minas Gerais. Ciência e Agrotecnologia, Lavras, v. 32, n. 2, p. 532-539, 2008. DOI: 70542008000200029

MELLO, C. R.; SILVA, A. M. da. Modelagem estatística da precipitação mensal e anual e no período seco para o estado de Minas Gerais. Revista Brasileira de Engenharia Agrícola e Ambiental, Campo Grande, v. 13, n. 1, p. 68-74, 2009. DOI: http://dx.doi.org/10.1590/S1415-43662009000100010

NAGHETTINI, M.; PINTO, E. J. A. Hidrologia Estatística [Statistical Hydrology]. 1. ed. Belo Horizonte: CPRM, 2007. $57 \mathrm{p}$.

OLIVEIRA, L. F. C. de; ANTONINI, J. C. dos A.; GRIEBELER, N. P. Estimativas de chuvas intensas para o Estado de Goiás. Engenharia Agrícola, Jaboticabal, v. 28 , n. $1, \quad$ p. $22-33, \quad 2008$. DOI: http://dx.doi.org/10.1590/S0100-69162008000100003

TUCCI, C. E. M. Modelos hidrológicos. Porto Alegre: UFRGS, 2000. 669 p.

VILLARINI, G.; SMITH, J. A.; BAECK, M. L.; VITOLO, R.; STEPHENSON, D. B.; KRAJEWSKI, W. F. On the frequency of heavy rainfall for the Midwest of the United States. Journal of Hydrology, Amsterdam, v. 400, n. 1, p. 103-120, 2011. DOI: https://doi.org/10.1016/j.jhydrol.2011.01.027 